\title{
Clinical and biochemical response to neridronate treatment in a patient with osteoporosis-pseudoglioma syndrome (OPPG)
}

\author{
M. Celli ${ }^{1}$ (I) - P. D'Eufemia ${ }^{1}$ - P. Persiani ${ }^{2}$ - A. Turchetti $^{1}$ - A. Febbo ${ }^{1} \cdot$ Y. D'Alfonso $^{1}$. \\ L. Celli ${ }^{1} \cdot$ A. Zambrano ${ }^{1}$
}

Received: 9 June 2017 / Accepted: 25 August 2017

(C) International Osteoporosis Foundation and National Osteoporosis Foundation 2017

\begin{abstract}
Osteoporosis-pseudoglioma syndrome (OPPG) is a rare autosomal recessive syndrome characterized by juvenileonset osteoporosis and ocular abnormalities due to a lowdensity lipoprotein receptor-related protein 5 (LRP5) gene mutation. Treatment with bisphosphonates, particularly with pamidronate and risedronate, has been reported to be of some efficacy in this condition. We report on a patient with OPPG due to an LRP5 gene mutation, who showed an encouraging response after a 36-month period of neridronate therapy. We report a case of a patient treated with bisphosphonates. Bisphosphonates should be administered in OPPG patients as a first-line therapy during early childhood.
\end{abstract}

Keywords Bisphosphonate $\cdot$ Bone $\cdot$ LRP5 $\cdot$ OPPG $\cdot$ Osteoporosis-pseudoglioma $\cdot$ Osteoporosis-pseudoglioma syndrome

\section{Introduction}

Osteoporosis-pseudoglioma syndrome (OPPG) is a rare autosomal recessive disorder characterized by severe early-onset osteoporosis and blindness [1-4] Typical OPPG manifestations include fractures and congenital blindness due to persistence of the fetal fibrovascular

M. Celli

m.celli@policlinicoumberto1.it

1 Department of Pediatrics, "Sapienza" University of Rome, Viale Regina Elena, 324, 00324 Rome, Italy

2 Department of Anatomic Histologic Forensic and Locomotor Apparatus Sciences, "Sapienza" University of Rome, Viale Regina Elena, 324, 00324 Rome, Italy ocular system and failure of retinal development. The phenotype is variable even among siblings [3]. Osteoporosis usually manifests in early childhood with recurrent long bone fractures, vertebral compression fractures and, reduced bone mineral density (BMD). More severely affected patients may have muscle weakness, bowing of the long bones, and severe spinal deformities [3-5]. The ocular phenotype ranges from vascularization abnormalities from congenital phthisis bulbi to milder vitreoretinal changes [1-3]. Mental development is usually normal, but almost $25 \%$ of patients show cognitive impairment [1]. OPPG syndrome is caused by homozygous mutations in the low-density lipoprotein (LDL) receptor-related protein 5 (LRP5) gene on chromosome 11q13.4 [2, 6].

Based on a few published case reports, treatment with bisphosphonates improves BMD in patients with OPPG syndrome [7-9].

The main effect of pharmacologically active bisphosphonates is to reduce bone turnover decreasing bone resorption. The molecular mechanism has been partially revealed. It was found that nitrogen-containing bisphosphonates prevent osteoclast function by inhibiting the mevalonate pathway, which is responsible for cholesterol and isoprenoid lipid production [10]. Neridronate is an amino-bisphosphonate that is structurally similar to alendronate and pamidronate. It only differs in the number of side-chain methyl groups and has been investigated in Paget bone disease [11] and osteogenesis imperfecta [12, 13].

We report the case of a patient with OPPG and a novel LRP5 mutation who received neridronate treatment for a 36month period. We show that neridronate therapy improves the lumbar spine bone mineral density Z-score, bone turnover markers, bone pain, and quality of life. 


\section{Case report}

A 16-year-old Tunisian boy presented with a suspected diagnosis of osteogenesis imperfecta because of limb, femur, radius and ulnar deformities, spinal compression fractures, reduced bone mineral density, and a COL1A1 gene heterozygotic mutation. His parents were first cousins, who were also sons of consanguineous parents, and were reported to have the same condition. Eleven additional siblings were said to be unaffected. No additional information about other family members, or, data on the neonatal period, were available. Psychomotor development was normal. The patient was completely blind, and his visual problems were noted at 10 months of age. At 6 years of age, left tibial bowing was noted, and right lower limb and spinal deformities, such as marked kyphosis, subsequently developed.

At the time of our first evaluation, the patient did not show limb fractures. Psychomotor development was normal. He had short stature $(<3$ rd percentile). Ligament laxity was evident in the lower limbs. The pupils were not reactive to light. The patient could stand unsupported, but he could walk only with support because of visual problems.

The ophthalmologic evaluation revealed bilateral cataracts, right microphthalmia, left retinal calcifications, and visual evoked potentials were absent. Abdominal and heart ultrasounds did not detect anomalies. The auditory brainstem response was in the normal range. Skeletal radiographies revealed generalized osteoporosis, scoliosis, dorsal kyphosis, and bowing and deformation of the lower limbs' long bones with a "serpentine" left fibula (Fig. 1). Spinal X-rays revealed severe dorsal vertebral fractures (Fig. 1) but only a mild reduction of the body height in the lumbar tract. Lumbar spine densitometry, performed by DXA (Hologic Discovery Densitometer 4500), detected a severely reduced bone mass (Table 1).

On the basis of clinical and instrumental findings, LRP5 gene analysis was performed and revealed a deletion involving exon 4 and part of introns 3 and 4, as previously described [14].

After a baseline evaluation of bone and mineral metabolism (Table 1), when the patient was 16 years old, intravenous treatment with neridronate was started in a single administration of $2 \mathrm{mg} / \mathrm{kg}$ and repeated every 3 months for a total period of 3 years. We also found a vitamin D deficiency $[25(\mathrm{OH}) \mathrm{D}$ : $9.5 \mathrm{ng} / \mathrm{mL}]$, and we started cholecalciferol and calcium administration at a daily dose of $800 \mathrm{UI}$ and $1000 \mathrm{mg}$, respectively. The efficacy of treatment was established by assessing the patient's bone pain, growth parameters, bone turnover markers, spine X-rays, and lumbar spine bone densitometry (Table 1). The numerical rating scale (NRS), a subjective psychometric response scale based on a numerical gradient from 0 (no pain) to 10 ("worst possible pain"), showed a significant reduction of bone pain ( $5 \mathrm{vs} 9$ initial evaluation) after 6 months of neridronate treatment; no pain was reported in the following visits. Serum osteocalcin (BGP), a bone formation marker, peaked at 12 months and remained higher compared with the baseline value. Collagen type $1 \mathrm{C}$-telopeptide (CTX) and bone-alkaline phosphatase (BALP), which are respectively bone resorption and formation markers, showed a significant reduction after 12 months of treatment and an additional slight reduction after 24 and 36 months, compared with the baseline levels. There were no changes in the calcium, phosphorus, or PTH serum levels. Before treatment, 25(OH)D was insufficient, but after a 12-month period of cholecalciferol administration at a daily dose of $800 \mathrm{UI}$, it became normal and remained within the normal range after 24 and 36 months of evaluation. A spinal X-ray was repeated at the end of the treatment and no new fractures were detected on radiographies during the 36-month period of treatment. Regarding lumbar spine mineral bone densitometry, the bone mineral content (BMC) and bone mineral density (BMD) were substantially below the normal values at presentation. During treatment, a substantial improvement in BMC and BMD was observed. The BMC had an increase of $35.5 \%$ by 1 year and from
Fig. 1 Radiographs of the spine revealed severe dorsal vertebral fractures (1a), and deformation of the long bones as "serpentine" left fibula (1b), before neridronate treatment

Table 1 Calcium metabolism parameters, bone turnover markers, and bone mineral density during neridronate treatment

\begin{tabular}{llllll}
\hline & T0 & 1 year & 2 years & 3 years & $p$ \\
\hline $\begin{array}{l}\text { Serum calcium (adjusted for serum } \\
\quad \text { albumin levels) (mg/dL) (n.v. 8.40-10.0) }\end{array}$ & 9.1 & 8.8 & 8.8 & 8.8 & n.s. $^{\text {a }}$ \\
$\quad$ Serum phosphate (mg/dL) (n.v. 2.80-4.60) & 3.4 & 3.5 & 3.3 & 3.4 & n.s. $^{\text {a }}$ \\
Intact PTH (pg/mL) (n.v. 10.6-54) & 46.9 & 43.4 & 44.6 & 43.7 & n.s. $^{\text {a }}$ \\
CTX (ng/mL) (n.v. 0.11-0.75) & 2.1 & 1.0 & 1.1 & 0.5 & $0.001^{\text {a }}$ \\
25-OH vit D (ng/mL) (n.v. > 32) & 9.5 & 28 & 32 & 32 & $0.01^{\text {a }}$ \\
BALP (U/L) (n.v. 15.0-41.3) & 190 & 73.7 & 65.2 & 63.1 & $0.01^{\text {a }}$ \\
Serum osteocalcin (ng/mL) (n.v. 24.0-70.0) & 21 & 74.8 & 76.43 & 78.2 & $0.01^{\text {a }}$ \\
Lumbar spine area (cm $\left.{ }^{2}\right)$ & 39.9 & 44.7 & 48.8 & 43.8 & $0.01^{\text {a }}$ \\
Lumbar spine BMC (g) & 17.5 & 23.8 & 29.4 & 29.7 & $0.01^{\text {a }}$ \\
Lumbar spine BMD (g/cm $\left.{ }^{2}\right)$ & 0.44 & 0.53 & 0.60 & 0.67 & $0.001^{\text {a }}$ \\
Lumbar spine BMD (Z-score) & -6.1 & -3.8 & -3.6 & -3.3 & $0.001^{\text {a }}$ \\
\hline
\end{tabular}

Significance $p<0.05 . p$ value represents the significance of the difference of results at the four time points determined using analysis of variance

${ }^{\text {a }}$ Significant difference to result at 3 years vs T0 (paired $t$ test)
67.7 to $69.4 \%$ by 36 months. The BMD Z-score was -6.1 at presentation, -3.8 after 1 year, -3.6 after 2 years, and -3.3 after 3 years ( $p<0.001$ paired $t$ test) (Table 1$)$. According to the normal values given by the manufacturers, we would have been expected a BMD increase of $45.9 \%$ during the entire 36month period.

Informed consent was obtained from the subject described in the study.

\section{Discussion}

The patient reported herein was diagnosed with a very rare form of juvenile-onset severe osteoporosis and blindness known as OPPG. The genetic data have been previously discussed [14].

To date, approximately 70 patients have been reported in literature. Bone fractures, vertebral compression fractures, BMD reductions, and ocular anomalies due to abnormal vascularization include retrolental masses, phthisis bulbi, microphthalmia, microcornea, clouding of the cornea and lens, intraocular hemorrhage, and retinal detachment are present in early childhood [7-9]. Alonso $\mathrm{N}$ et al. described the first case of atypical subtrochanteric femoral fracture in an OPPG patient with two novel LRP5 mutations [15].

Homozygous or compound heterozygous loss-of-function mutations of the LRP5 gene cause OPPG [2]. LRP5 acts as a transmembrane coreceptor in the Wnt signaling pathway, which is involved in the regulation of osteoblast growth and differentiation. LRP5 acts in gut cells, not in osteoblasts, to control bone formation via a Wnt-independent pathway and identifies a new hormone, serotonin, and a novel endocrine axis regulating bone mass, with important therapeutic implications [16]. LRP5 anomalies are known to cause recessive familiar exudative vitro-retinopathy [17] and an autosomal dominant syndrome with high bone density, a wide and deep mandible, and torus palatinus.

Few open-label studies describe therapeutic options in OPPG patients. In particular, the efficacy of bisphosphonates has been reported in 12 patients overall [7, 9]. Zacharin and Cundy first reported in 2000 the effect of a 2-year administration of bisphosphonate (pamidronate in two cases and clodronate in one case). The authors reported clinical improvement in terms of (a) reduction of bone pain within the first month of treatment, (b) lower morbidity especially after 6 months, (c) no detection of new fractures on radiography during the entire 2-year period of treatment, and (d) improvements in vertebral height and end plate remodeling. Some authors assert that bone resorption markers showed an initial suppression during the first 3 to 6 months of treatment in all 3 subjects evaluated, but the levels subsequently increased. The same pattern was observed for osteocalcin but not for alkaline phosphatase [8].

Similar results using pamidronate were shown by Di Iorgi $\mathrm{N}$ [18] and Bayram F et al. in 2006 [19].

Subsequently, Streeten EA and colleagues [7] described improvement in the BMD Z-score in OPPG patients after bisphosphonates administration and with the osteoanabolic drug teriparatide after stopping the bisphosphonates. They reported a beneficial response to bisphosphonates in 4 patients and the lack of a definitive response to teriparatide in 1 patient. This finding suggested the initiation of bisphosphonates treatment in OPPG patients. Barros ER et al. reported a 3-year follow-up of pamidronate therapy in two brothers with OPPG. He observed an increased BMD and decreased fracture rate [20].

In 2011, Arantes HP. et al. described a patient treated for 2 years with teriparatide [21]. Over 2 years of treatment, the 
BMD markedly increased in the lumbar spine and in the right total hip. The CTx level reached a peak by 3 months and slowly returned to the baseline level by 24 months.

Bisphosphonates primarily work by inhibiting osteoclastic bone resorption. In our patient, this occurrence was reflected by an early reduction in bone resorption markers. Whether bisphosphonates influence the basic defect in OPPG is not clear at present but an important component of their efficacy may be their action to reduce bone pain and bone loss due to immobility. The neridronate effects on spinal bone density observed in our patients were similar to those described for other bisphosphonates, administered either orally or intravenously, in different forms of childhood osteoporosis [12, 13]. In conclusion, our results in terms of increased bone mineral density, pain reduction, and better quality of life suggest that neridronate is an alternative treatment to other bisphosphonates already described.

\section{Compliance with ethical standards}

Conflicts of interest None.

\section{References}

1. Ai M, Heeger S, Bartels CF, Schelling DK (2005) Clinical and molecular findings in osteoporosis-pseudoglioma syndrome. Am J Hum Genet 77:741-753

2. Gong Y, Vikkula M, Boon L (1996) Osteoporosis-pseudoglioma syndrome, a disorder affecting skeletal strength and vision, is assigned to chromosome region 11q12-13. Am J Hum Genet 59: 146-151

3. Somer H, Palotie A, Somer M, Hoikka V, Peltonen L (1988) Osteoporosis-psedudoglioma syndrome: clinical, morphological and biochemical studies. J Med Genet 25:543-549

4. Frontali M, Dallapiccola B (1986) Osteoporosis-pseudoglioma syndrome and the ocular form of osteogenesis imperfect. Clin Genet 29:262

5. Lev D, Binson I, Foldes AJ, Watemberg N, Lerman-Sagie Y (2003) Decreased-bone density in carriers and patients of an Israeli family with the osteoporosis-pseudoglioma syndrome. Isr Med Assoc J 5: 419-421

6. Gong Y, Slee RB, Fukai N, Warman ML (2001) Osteoporosispseudoglioma syndrome collaborative group: LDL receptorrelated protein 5 (LRP5) affects bone accrual and eye development. Cell 107:513-523

7. Streeten EA, McBride D, Puffenberger E, Hoffman ME, Pollin TI, Donelly P, Sack P, Morton H (2008) Osteoporosis-pseudoglioma syndrome: description of 9 new cases and beneficial response to bisphosphonates. Bone 43:584-590

8. Zacharin M, Cundy T (2000) Osteoporosis pseudoglioma syndrome: treatment of spinal osteoporosis with intravenous bisphosphonates. J Pediatr 137:410-415

9. Levasseur R (2008) Treatment and management of osteoporosispseudoglioma syndrome. Expert Rev Endocrinol Metab 3:337-348

10. Gatti D, Rossini M, Viapiana O, Idolazzi L, Adami S (2013) Clinical development of neridronate: potential for new applications. Ther Clin Risk Manag 9:139-147

11. Adami S, Bevilacqua M, Broggini M, Filipponi P, Ortolani S, Palummeri E, Ulivieri F, Nannipieri F, Braga V (2002) Short-term intravenous therapy with neridronate in Paget's disease. Clin Exp Rheumatol 20:55-58

12. Gatti D, Antoniazzi F, Prizzi R, Braga V, Rossini M, Tatò L, Viapiana O, Adami S (2005) Intravenous neridronate in children with osteogenesis imperfect: a randomized controlled study. J Bone Miner Res 20:758-763

13. D'Eufemia P, Finocchiaro R, Zambrano A, Lodato V, Celli L, Finocchiaro S, Persiani P, Turchetti A, Celli M (2017) Serum creatine kinase isoenzymes in children with osteogenesis imperfect. Osteoporos Int 28:339-346

14. Laine CM, Chung BD, Susic M, Prescott T, Semler O, Fiskerstrand D'EP, Castori M, Pekkinem M, Sochett E, Cole WG, Netzer C, Makitie O (2011) Novel mutations affecting LRP5 splicing in patients with osteoporosis-pseudoglioma syndrome (OPPG). Eur J Hum Genet 19:875-881

15. Alonso N, Soares DC, McCloskey EV, Summers GD, Raltston SH, Gregson CL (2015) Atypical femoral fracture in osteoporosis pseudoglioma syndrome associated with two novel compound heterozygous mutations in LRP5. J Bone Miner Res 30(4):615-620

16. Yadav VK, Ducy P (2010) LRP5 and bone formation. A serotonindependent pathway. Ann N Y Acad Sci 1192:103-109

17. Toomes C, Bottomley HM, Jackson RM et al (2004) Mutations in LRP5 of FZD4 underlie the common familial exudative vitreoretinopathy locus on chromosome 11q. Am J Hum Genet 74:721-730

18 Di Iorgi N, Maghnie M (2006) Motor function improvement after intravenous pamidronate in osteoporosis pseudoglioma syndrome. J Pediatr 149:734

19 Bayram F, Tansiverdi F, Kurtoglu S, Atabek ME, Kula M, Kaynar L, Kelestimur F (2006) Effects of 3 years of intravenous pamidronate treatment on bone markers and bone mineral density in a patient with osteoporosis-pseudoglioma syndrome (OPPG). J Pediatr Endocrinol Metab 19:275-279

20 Barros ER, Dias da Silva MR, Kunii IS, Lazaretti-Castro M (2008) Three years follow-up of pamidronate therapy in two brothers with osteoporosis-pseudoglioma syndome (OPPG) carrying an LRP5 mutation. J Pediatr Endocrinol Metab 21:911

21 Arantes HP, Barros ER, Kunii I, Bilezikian JP, Lazaretti-Castro M (2011) Teriparatide increases bone mineral density in a man with osteoporosis pseudoglioma. J Bone Miner Res 26:2823-2826 